\title{
Regenerative spiking oscillation in a semiconductor laser with a nonlinear delayed feedback
}

\author{
Yun Liu \\ Graduate School of Electronic Science and Technology, Shizuoka University, 3-5-1 Johoku, Hamamatsu 432, Japan \\ Junji Ohtsubo \\ Faculty of Engineering, Shizuoka University, 3-5-1 Johoku, Hamamatsu 432, Japan
}

(Received 19 October 1992)

\begin{abstract}
Routes to regenerative spiking periodic oscillations are discussed in a delayed-feedback optical bistable system consisting of a laser diode interferometer. The observed oscillations have almost the same fundamental period as the delay time or the period of its subharmonics. The dependence of bifurcation routes on the delay time and other control parameters is investigated. The linear stability analysis of the dynamical equation is conducted. The obtained mode distributions show good agreements with the spectral distributions of these spiking oscillations. Numerical simulations for several steady states are also given. They show that such spiking oscillations indeed exist in certain parameter regions.
\end{abstract}

PACS number(s): 42.50.Lc, 42.60.Mi

\section{INTRODUCTION}

In the past few years, instabilities and chaos have been explored extensively in a wide variety of quantum optical systems [1]. Not long after the invention of the laser, it was found that the laser is a good device to investigate not only coherence properties of light but also nonlinear dynamics such as deterministic chaos. The study of chaos in a laser system was motivated by Haken [2], who analyzed the physics of single-mode lasers and pointed out the similarity between Maxwell-Bloch equations and Lorenz equations, which is known as a typical lowdimensional chaotic model [3]. However, the large difference of the damping times among lasing parameters, i.e., the damping times of the electric field, the polarization, and the population inversion, eliminates the possibility of observing chaos in most of available lasers. The subject had not attracted much attention until Ikeda described an optical instability in a ring resonator containing a two-level medium with a period-doubling cascade leading to a chaotic output [4]. Since then, similar phenomena, including bifurcation scenarios and some universal properties of optical chaos, have been predicted in a wide class of passive optical systems with a feedback loop. The first experimental demonstration was done in a hybrid system with an electronic feedback [5]. A demonstration of an all-optical system of the Ikeda instability appeared a little later [6]. Up to now, most experimental demonstrations have been performed by using hybrid systems because of their experimental simplicity, good agreement with theoretical models, and the suitableness for observations of the Ikeda instability, which requires that the response time of the nonlinear medium be short enough to allow a period- $2 t_{r}$ oscillation (where $t_{r}$ is the delay time of the system).

From a theoretical point of view, dissipative systems with delayed feedback are usually described by a delayed differential equation,

$$
\frac{d \mathbf{X}(t)}{d t}=\mathbf{F}\left(\mu ; \mathbf{X}(t), \mathbf{X}\left(t-t_{r}\right)\right)
$$

where $\mathbf{X}$ is a vector variable, $\mu$ is a control parameter, and $\mathbf{F}$ is a nonlinear function including dissipative effects. The solutions of the above equation exist in an infinitedimensional phase space, since the initial condition is specified on the whole interval $\left(0, t_{r}\right)$.

Generally, the system described by Eq. (1) consists of two factors: the discrete property characterized by the delay time $t_{r}$, and the continuous property represented by the differential term. Although most phenomena in a nonlinear system, such as periodic cycles, bifurcation scenarios, and higher-mode solutions, can be explained quite well based on the discrete property, the complex dynamics of the system can be fully understood by its continuous property [7]. An early study of the effects of the continuous property was made by the groups of Narducci and Mandel [8,9]. In Refs. [10-13], it was shown that the continuous property of the system plays a very important role in describing some complex dynamical behaviors of nonlinear systems.

The present authors have proposed a hybrid system which consists of a laser diode Twyman-Green interferometer with a delayed optoelectronic feedback loop [14]. One of the main features in our system is the easy control of the parameters involved in the chaotic system. This advantage facilitates our observations of some novel oscillations related to frequency-locked wave forms and the period 3 cycle [12]. Recently, we reported the observation of spiking oscillations that we called the period-1 oscillations, since the period of the oscillations is almost the same as the delay time [15]. To our knowledge, this is the first observation of such oscillations in a delayed optical bistable system with a large delay time.

This paper concerns a further study about the spiking oscillations in the delayed-feedback hybrid bistable system with a large delay time. In Sec. II, the experimental 
model of our system is briefly described and a set of the coupled equations is obtained. Section III presents the experimental observations of regenerative spiking oscillations. The oscillations having the same period as the delay time and the period of its subharmonics are obtained. The dependence of the oscillations on the control parameters is also investigated. The theoretical analysis and numerical simulations are given in Sec. IV. By use of the steady-state assumption, the linear mode analysis of the system is performed under various parameter conditions. Comparison between the mode distributions and the spectral distributions of the spiking oscillations is also made, and the result shows good agreement between them. The numerical calculations represent the evolution routes to chaos for the different steady states and coincide with the experimental results.

\section{EXPERIMENTAL ARRANGEMENT AND ITS DYNAMICAL MODEL}

The experimental setup of our system is shown in Fig. 1. The light source (Hitachi HL7801E single-mode AlGa-As channel-substrate planar laser diode) is powered by a current-driving circuit. By using such a circuit, the bias injection current is controlled up to the minimum resolution of $0.02 \mathrm{~mA}$. The temperature is stabilized with $0.02{ }^{\circ} \mathrm{C}$ by a temperature control device and the modulation for the injection current is small, so that the mode hop of the laser can be ignored during the experiment. An optical isolator with more than a $30-\mathrm{dB}$ isolation is used to avoid unwanted back-reflections into the laser diode. Then, the laser beam is expanded and directed into a Twyman-Green interferometer. The main role of the interferometer is to convert the frequency variation of the light source into the phase variation by the nonlinear interference signal. The interference signal is detected by a photodiode through a pinhole, being much smaller than the interference fringe separation. The detected signal is amplified and fed back into the injection current of the laser diode. A time lag is introduced in the feedback loop and the delay time is controlled by a computer within the range of $1 \mu \mathrm{s}-4 \mathrm{~ms}$. The light output power is measured through a built-in photodiode within the laser diode package and the detected signal is

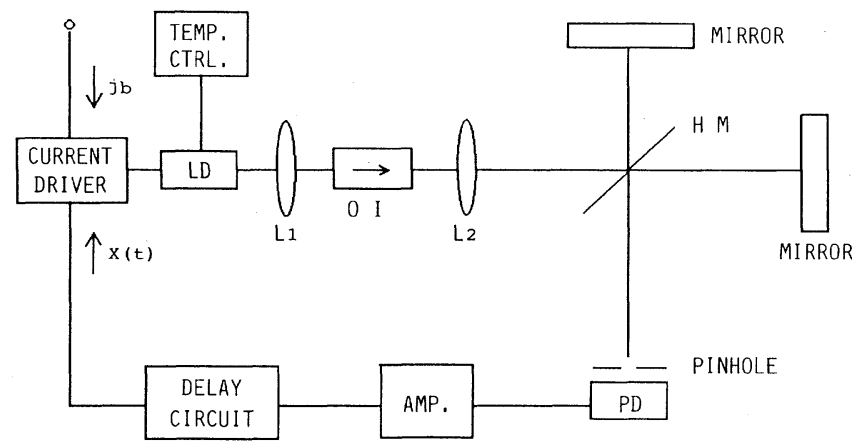

FIG. 1. Experimental setup. LD, laser diode; PD, photodiode; OI, optical isolator; L1 and L2, lenses; HM, half mirror. fed to a digital oscilloscope, and then is sent to the computer for the numerical analysis.

The key elements in our system are the laser diode and the interferometer. The variation of the injection current gives rise to the changes of the laser output power and the oscillation frequency with linear relations as $\delta P \propto \delta j_{b}$ and $\delta f \propto \delta j_{b}$, where $\delta P, \delta f$, and $\delta j_{b}$ are the changes of the output power, the oscillation frequency, and the injection current, respectively. On the other hand, these changes result in the intensity variations of the interference signal at the detection point and the variations of the injection current itself. The important point is that the intensity of the output of the interferometer changes nonlinearly with the laser oscillation frequency and, accordingly, this relation forms the nonlinearity of the whole system.

The dynamics of the laser oscillation is governed by the rate equations

$$
\begin{aligned}
& \frac{d P(t)}{d t}=\Gamma g N_{t h} \tau_{s}\left[n(t)-n_{0}\right] P(t)-\gamma_{p}^{-1} P(t), \\
& \frac{d n(t)}{d t}=j_{b}-x(t)-n(t)-g \xi^{1} \tau_{s}\left[n(t)-n_{0}\right] P(t),
\end{aligned}
$$

where $x(t)$ is the contribution of the feedback loop and is subject to the delayed differential equation

$$
\gamma_{t} \frac{d x(t)}{d t}=-x(t)+f\left[P\left(t-t_{d}\right)\right],
$$

with the nonlinear function [14]

$$
f[P(t)]=\mu P(t)\left\{1+b \cos \left[\kappa P(t)-\phi_{0}\right]\right\} .
$$

Here $P$ is the laser output power, $n$ is the carrier density normalized by the threshold value $N_{\text {th }}, x$ is the delayed feedback current, $n_{0}$ is the carrier density at the transparency, $g$ is the linear gain coefficient, $\Gamma$ is the confinement factor, $\xi$ is the proportional coefficient of the laser intensity to the photon density and $j_{b}$ is the normalized pump with $j_{b}=1$ at the laser threshold. The time scale of the rate equations and the delayed differential equation is normalized by the carrier life time $\tau_{s}$. The parameters $\gamma_{p}, \gamma_{t}$, and $t_{d}$ represent the normalized values of the photon lifetime $\tau_{p}$, the response time $\tau$ of the feedback loop, and the delay time $t_{r}$, respectively, i.e., $\tau_{p}=\gamma_{p} \tau_{s}, \tau=\gamma_{t} \tau_{s}$, and $t_{r}=t_{d} \tau_{s}$. The parameter $\mu$ is the measure of the amplification for the light output power in one open feedback loop and can be controlled by the feedback gain, $\kappa$ is a parameter proportional to the optical path difference of the interferometer arms, and $b$ and $\phi_{0}$ are the constants in the interferometer.

\section{EXPERIMENTAL RESULTS}

In the experiment, we selected the parameters $\mu, j_{b}, \kappa$, and $t_{r}$ at appropriate values in order to obtain steadystate signals for different periodic cycles. For this purpose, we fixed $\mu$ at about 0.4 and the optical path difference of the interferometer arms to be $0.9 \mathrm{~cm}$, which corresponds to $\kappa=4.8 \pi \mathrm{mW}^{-1}$.

By varying the bias injection current to the laser diode within a certain current region, we observed two typical 


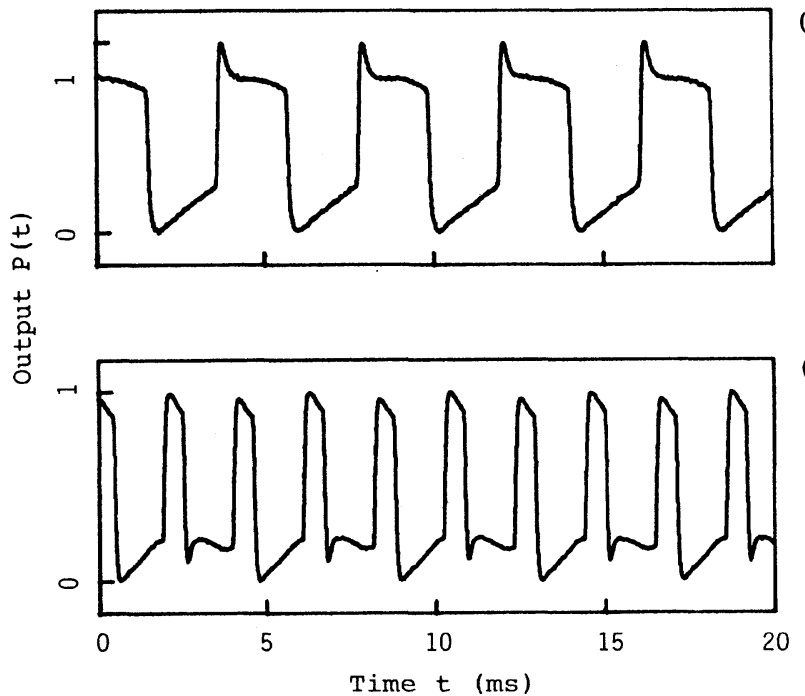

FIG. 2. Period-2 cycle and spiking oscillation for $t_{r}=2 \mathrm{~ms}$. Periods are (a) 4.08 and (b) 2.04 ms.

periodic cycles. Figure 2 shows typical periodic wave forms at the delay time of $2 \mathrm{~ms}$. Figure 2(a) corresponds to a period- 2 cycle with the period $4.08 \mathrm{~ms}$. By the slight change of the bias injection current, the period-doubling bifurcation scheme was observed. An abrupt change of the dynamical behavior was seen at a certain threshold current and, above the threshold, the dynamical wave form behaved differently from a normal bifurcation scheme. Beyond this critical current, the spiking oscillations, such as those shown in Fig. 2(b), were observed. The period of the wave form was $2.04 \mathrm{~ms}$, which has almost the same period of the delay time. By varying the bias injection current within a small range, we investigated the dependence of the dynamical behaviors of such spiking oscillations on the control parameters. Figure 3 shows four examples of spiking oscillations for the increase of the bias injection current. The successive change of the duty ratio of the squared wave can be seen with the notable deformation of the wave form. Such variations can be conveniently understood from the spectral distributions, which are discussed in the next section.

It should be noted that the spiking oscillations were observed within several regions of the bias injection current that are separated from other bifurcation regions. Here, in terms of normal bifurcation regions, we mean the regions where we can observe the usual perioddoubling bifurcations, intermittent bifurcations, periodic cycles, or chaotic outputs as we reported in our previous works.

By choosing both the delay time and the bias injection current in an appropriate range of the spiking oscillations, we investigated the successive evolutions of the output light intensity. Figure 4 shows two examples of them. The delay times for Figs. $4(a)$ and $4(b)$ were 0.5 and $1 \mathrm{~ms}$, respectively. The periods for the envelope and fine-structure components in Fig. 4(a) are 11.7 and 0.56 $\mathrm{ms}$, respectively. Meanwhile, the wave form in Fig. 4(b) has nearly the same period of the delay time.

\section{THEORETICAL ANALYSIS AND DISCUSSION}

\section{A. Linear stability analysis}

The oscillation mode near the threshold of the instability can be obtained by a linear stability analysis. The stead-state solutions of Eq. (2) are given by

$$
\begin{aligned}
& \bar{P}=\Gamma \xi \gamma_{p} N_{\mathrm{th}}\left(j_{b}-\bar{n}-\bar{x}\right), \\
& \bar{n}=n_{0}+\frac{1}{g \tau_{p} N_{\mathrm{th}}}, \\
& \bar{x}=f(\bar{P}),
\end{aligned}
$$

where $\bar{P}, \bar{n}$, and $\bar{x}$ are the steady-state values of the laser output power, the normalized carrier density, and the feedback current, respectively. Linear stability analysis is performed by the following assumption in Eq. (3):
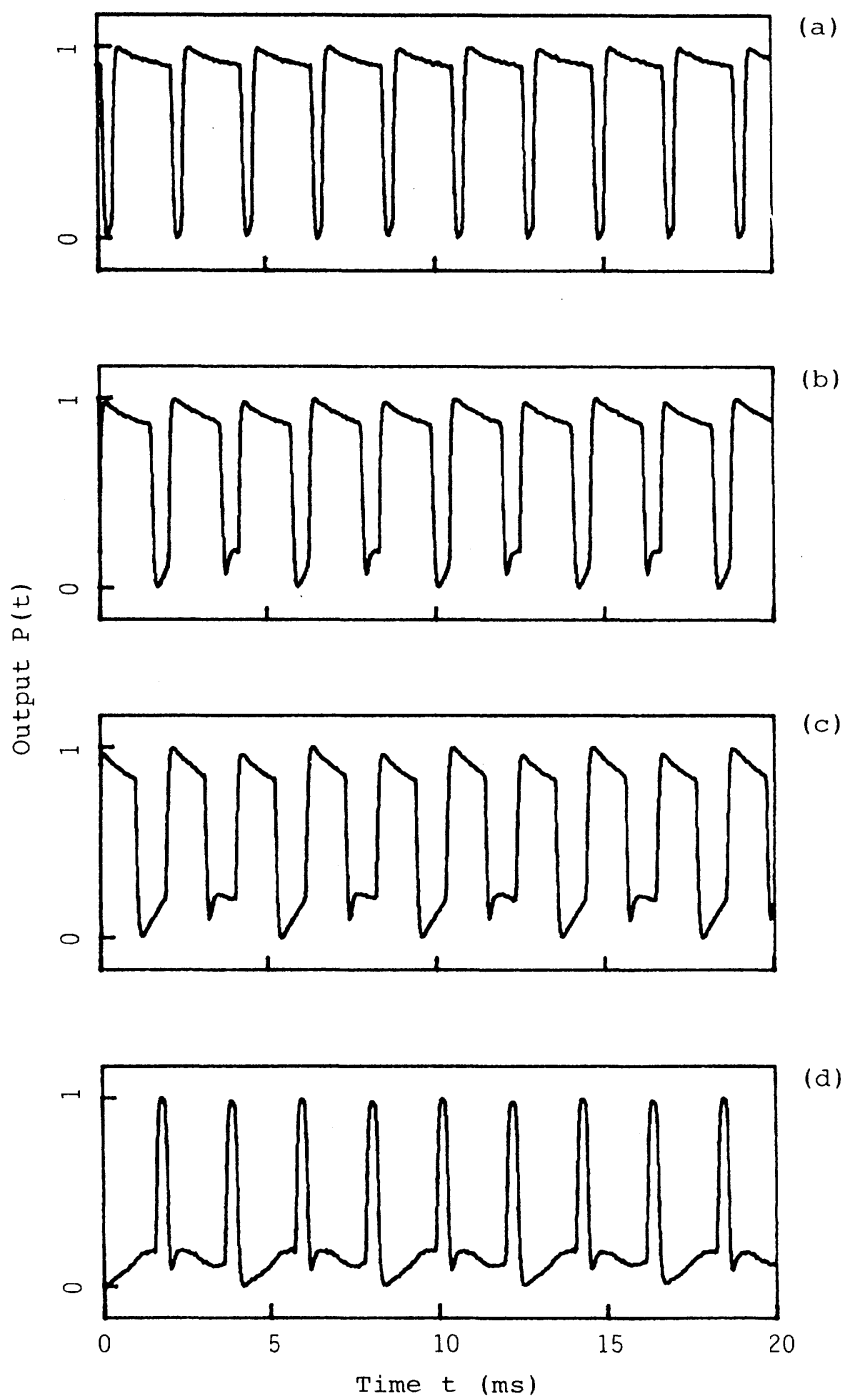

(d)

FIG. 3. Bifurcation routes of spiking oscillations at $t_{r}=2.0$ ms. The bias injection currents are (a) 44.32, (b) 44.46, (c) 44.68, and (d) $45.34 \mathrm{~mA}$. 

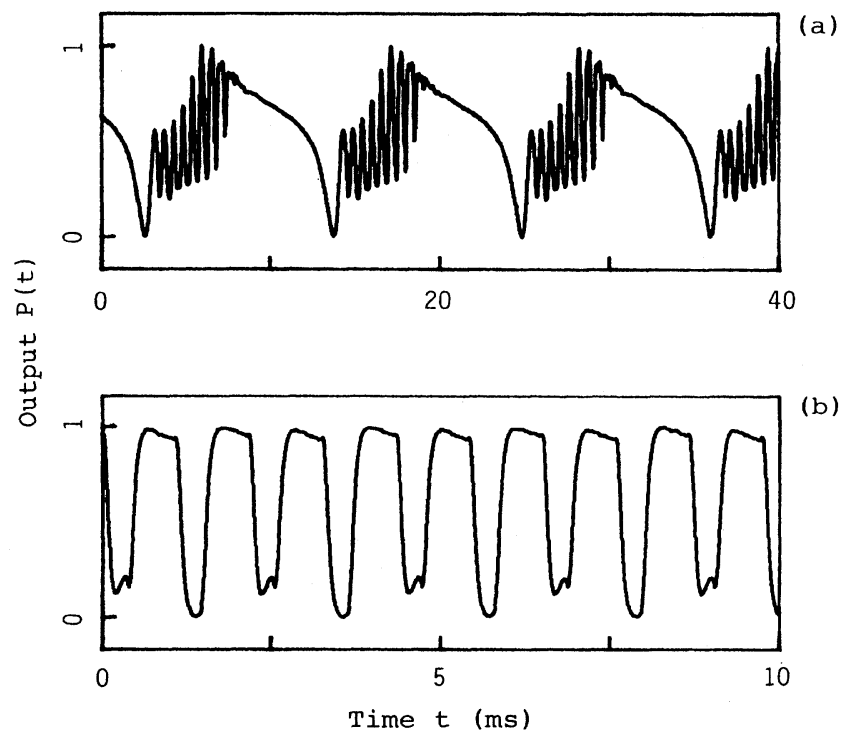

FIG. 4. (a) Relaxationlike oscillation at $t_{r}=0.5 \mathrm{~ms}$ and (b) period-1 oscillation at $t_{r}=1.0 \mathrm{~ms}$.

$$
z=\bar{z}+\overline{\delta z} \exp (\lambda t) \quad(z=P, n, x) .
$$

The characteristic equation then reads

$$
\lambda^{3}+A \lambda^{2}+B \lambda+C+D \exp \left(-\lambda t_{d}\right)=0,
$$

where

$$
\begin{aligned}
& A=1+\gamma_{t}^{-1}+A_{0}, \\
& B=\left(1+A_{0}\right) \gamma_{t}^{-1}+A_{0} \gamma_{p}^{-1}, \\
& C=A_{0}\left(\gamma_{t} \gamma_{p}\right)^{-1}, \\
& D=\Gamma g N_{\mathrm{th}} \tau \gamma_{t}^{-1} \bar{P} D_{f}, \\
& A_{0}=g \xi^{-1} \tau_{s} \bar{P} \\
& D_{f}=\left.\frac{d f[P(t)]}{d t}\right|_{P(t)=\bar{P}} .
\end{aligned}
$$

Equation (5) can be solved numerically. Each eigenvalue $\lambda_{i}$ ( $i$ being integer number) corresponds to a linear mode in which the real part determines the stability and the imaginary part controls the oscillation frequency of the mode. Some features in hybrid bistable systems can be derived from the nonlinear coupling among the modes. A good correspondence between the imaginary part of the mode and the spectral peaks of the wave form is obtained, which appeared in a wide variety of periodic and quasiperiodic oscillations and chaos $[10,16]$. In the following, the spectral structure of the experimentally observed spiking oscillations is explained based on the mode distributions. Under a certain condition of the system, the mode distribution strongly depends on both the steady state and the delay time. The steady-state condition is determined by the relation

$$
\bar{P}=P_{b}-\Gamma \xi N_{\mathrm{th}} \gamma_{p} f(\bar{P}),
$$

where $P_{b}=\Gamma \xi N_{\mathrm{th}} \gamma_{p}\left[j_{b}-n_{0}-\left(\Gamma g N_{\mathrm{th}} \tau_{p}\right)^{-1}\right]$ represents the bias output power of the laser diode. Equation (6) can be rewritten as the equilibria of the mapping by

$$
P_{n+1}=P_{b}-\Gamma \xi N_{\mathrm{th}} \gamma_{p} f\left(P_{n}\right)=f_{1}\left(P_{n}\right) .
$$

Since the above mapping contains a multimodal function,

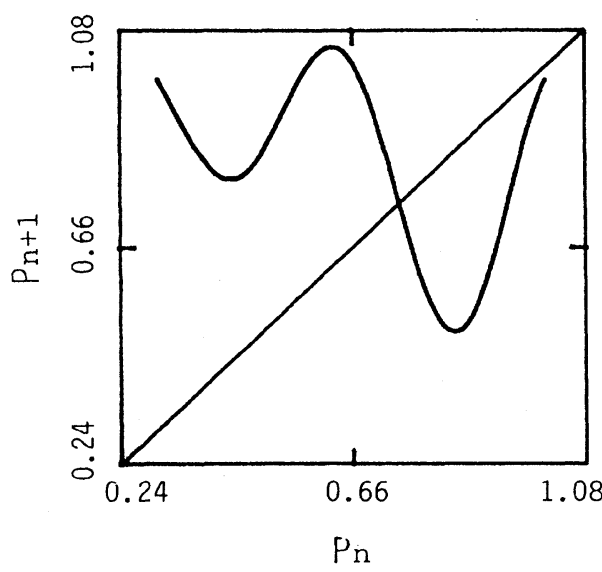

(a)
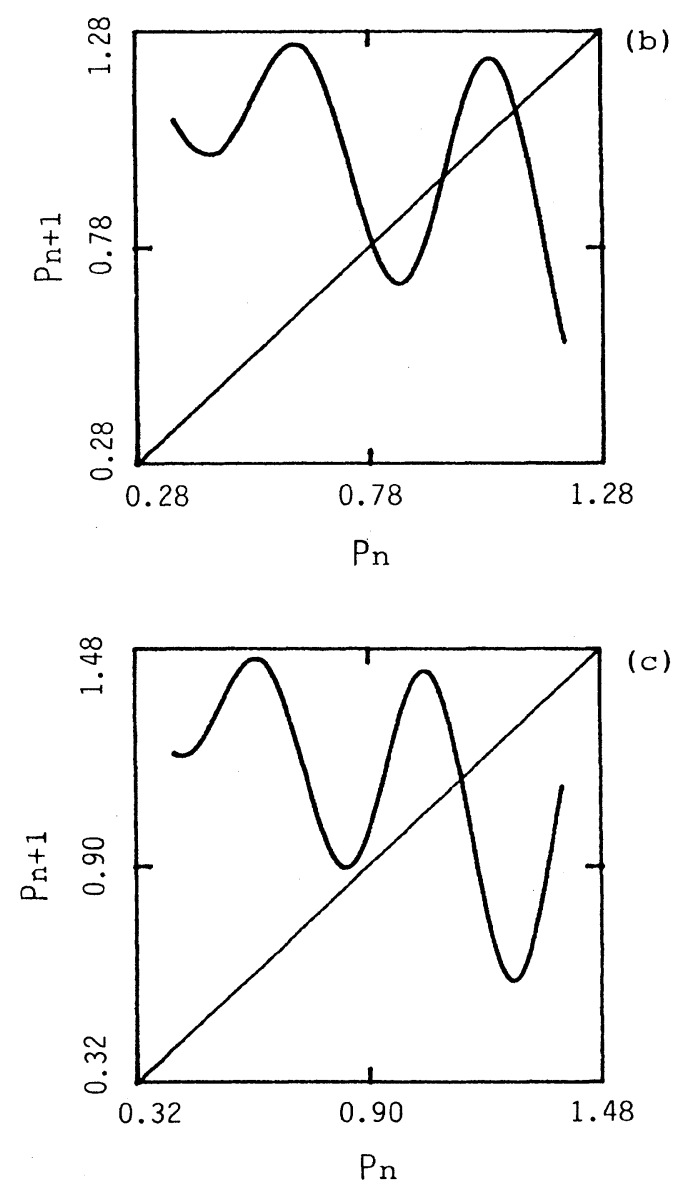

FIG. 5. Equilibria for the bias injection current of (a) $j_{b}=1.27$, (b) $j_{b}=1.32$, and (c) $j_{b}=1.37$. 
multiple equilibria may be present under a certain condition. Three examples of the equilibria for different bias injection currents are shown in Fig. 5. The parameter values were chosen as $\mu=0.4, \kappa=4.8 \pi \mathrm{mW}^{-1}, \phi_{0}=0$, $b=0.8, \quad N_{\mathrm{th}}=2.5 \times 10^{24} \mathrm{~m}^{-3}, g=1.2 \times 10^{-12} \mathrm{~m}^{3} \mathrm{~s}^{-1}$, $\xi=5.38 \times 10^{-21} \mathrm{~m}^{3} \mathrm{~mW}, \tau_{s}=2.0 \mathrm{~ns}, \gamma_{t}=1.0 \times 10^{4}$, $\gamma_{p}=1.0 \times 10^{-3}, \Gamma=0.3$, and $n_{0}=0.4$. These values are compatible with the actual experimental condition. Three equilibria can be seen in Fig. 5(b), whereas only one equilibrium exists in Figs. 5(a) and 5(c).

We specialize our calculations to the above three cases and obtain a set of the mode distributions for each equilibrium. Some lowest-mode solutions are plotted in Fig. 6. The notable features of these figures can be summarized as follows. In Fig. 6(a), which corresponds to the case of the single equilibrium in Figs. 5(a) and 5(c), the fundamental mode has the frequency about $1 / 2 t_{d}$ and each harmonic component takes an odd-integer multiple of the fundamental frequency. The mode distributions are also plotted in Fig. 6(b) for the upper and lower equilibria shown in Fig. 5(b). The circle and triangle symbols are the results of the modes which have the same tendency as the mode distributions in Fig. 6(a). However, the mode distribution corresponding to the middle equi-

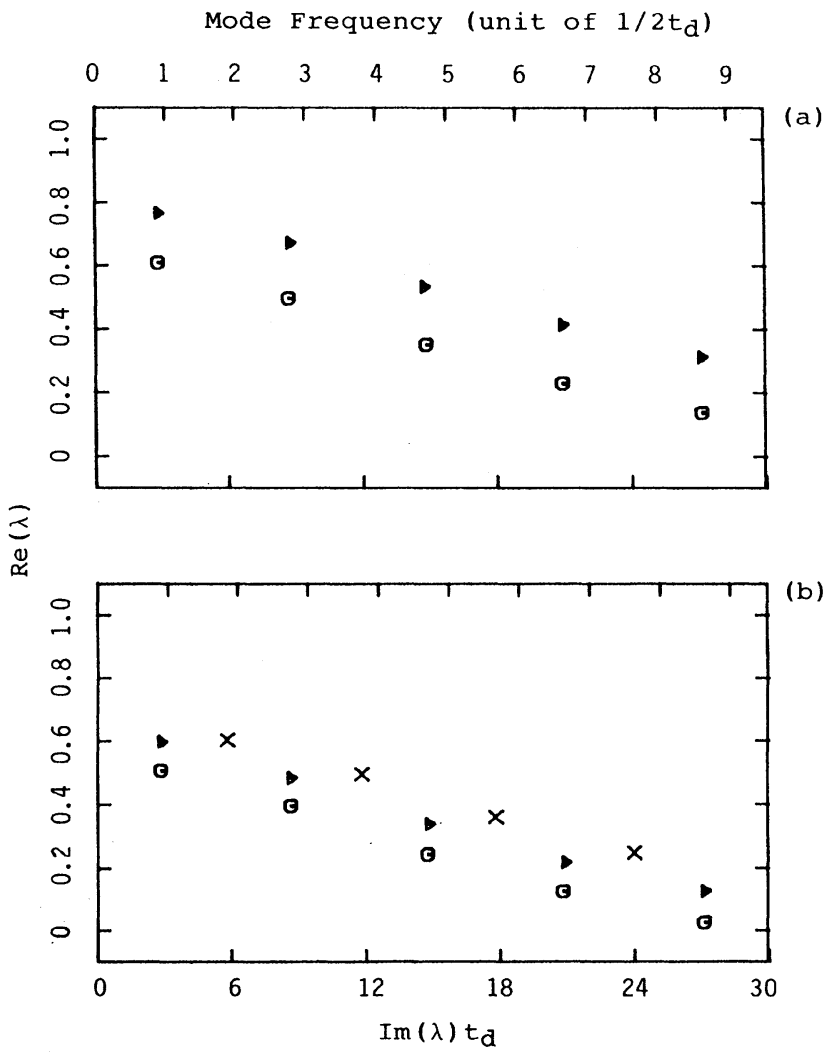

FIG. 6. Mode distributions corresponding to Fig. 5. (a) The case of a single equilibrium: $\bigcirc, \bar{P}=0.74 \mathrm{~mW}$ at $j_{b}=1.27$ corresponding to Fig. $5(\mathrm{a})$; and $\triangle, \bar{P}=1.13 \mathrm{~mW}$ at $j_{b}=1.37$ corresponding to Fig. 5(c). (b) The case of multiple equilibria: $O$, $\bar{P}=0.78 \mathrm{~mW} ; \times, \bar{P}=0.95 \mathrm{~mW} ;$ and $\triangle, \bar{P}=1.09 \mathrm{~mW}$ at $j_{b}=1.32$ corresponding to Fig. 5(b). librium in Fig. 5(b) displays a different behavior. As plotted in Fig. 6(b) by a crossed mark, the fundamental mode has the frequency near $1 / t_{d}$ and each harmonic component takes an integer multiple (not an odd-integer multiple) of the fundamental frequency.

\section{B. Comparison between mode and spectral distributions}

A comparison between the mode distributions and the power spectra of the experimental results is made. Figure 7 shows the power spectral distributions corresponding to the wave forms in Fig. 2. The upper scale in the figure represents the frequency normalized by a unit equal to $1 / 2 t_{d}$. In Fig. $7(\mathrm{a})$, the fundamental peak of the spectral frequency is calculated as $0.244 \mathrm{kHz}$. This value is about a half of $1 / t_{d}$. The dominant higher harmonic peaks appear at the positions corresponding to oddinteger multiples of the fundamental frequency. Meanwhile, in Fig. 7(b) the fundamental frequency peak is located at $0.488 \mathrm{kHz}\left(\sim 1 / t_{d}\right)$, and the peaks of its higher harmonics are integer multiples of the fundamental one, though weak subpeaks for the fundamental and its higher harmonics corresponding to the period $2 t_{d}$ can be seen. The phenomenon agrees quite well with the results obtained by the mode analysis in Fig. 6(b).

The spectral distributions corresponding to Fig. 3 are shown in Fig. 8. The relation between the fundamental frequency peak and its higher harmonics remain unchanged in Fig. 8. Although the subpeaks corresponding to a half of the fundamental frequency grow as the increase of the bias injection current, the variations of the bias injection current have little effect on the total spectral distributions for the evolution of the spiking oscillations.

It is well known that a periodic cycle in chaotic routes has its higher harmonic solutions corresponding to the higher modes. On the analogy of normal chaotic scenarios, one may expect the existence of harmonic solutions of some integer orders for the spiking oscillations. We show an example of the second mode solutions of the

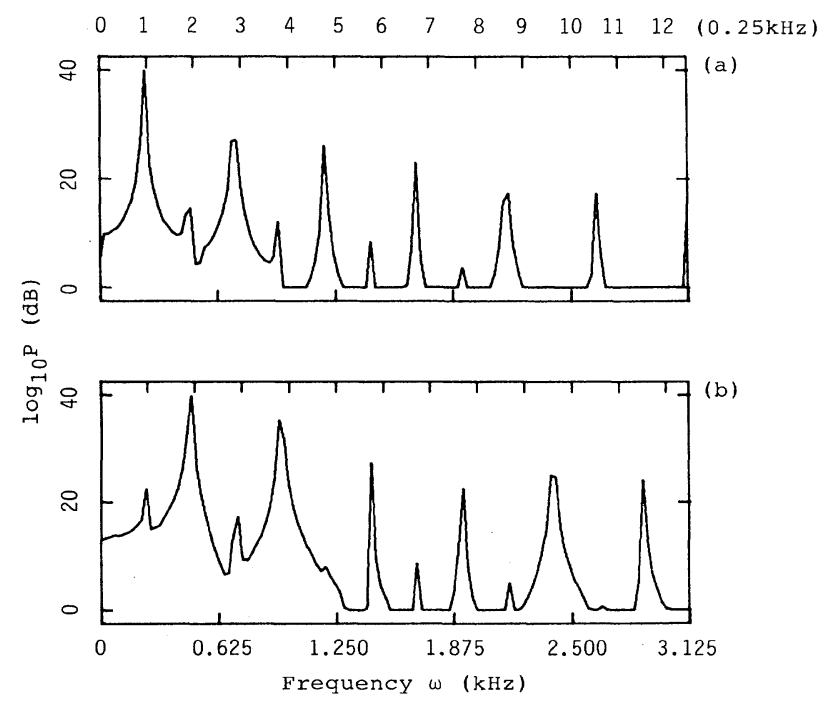

FIG. 7. Power spectral distributions corresponding to Fig. 2. 
spiking oscillations in Fig. 9. The frequency is computed as $1.8 \mathrm{kHz}$, which is nearly twice the frequency of $1 / t_{d}$.

\section{Numerical simulations}

We have employed the mode distributions to make a reasonable explanation for the spectral structure of the spiking oscillations. However, two questions still remain: (i) Do the substantial differences of the dynamics in the output power really exist for different parameter conditions, e.g., those shown in Figs. 5(a) and 5(b)? (ii) How does the system "select" a certain equilibrium among many existing equilibria for a given parameter conditions? To answer these questions, we numerically calculated Eq. (2) and investigated the evolution routes of the output power for different parameter conditions. The second-order Runge-Kutta algorithm was employed in the simulation. In order to eliminate the dependence on the initial conditions which inevitably emerges in the nu-
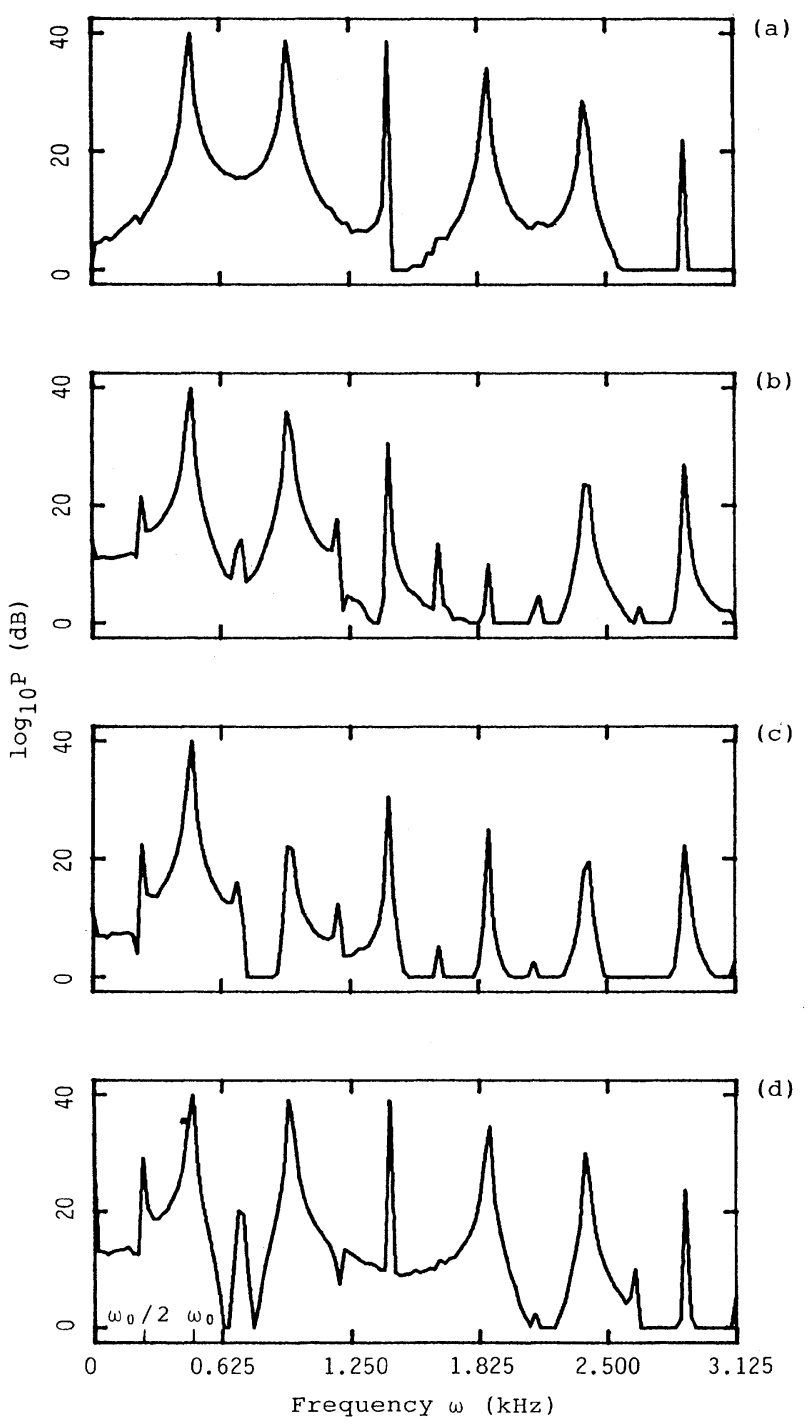

FIG. 8. Power spectral distributions corresponding to Fig. 4.

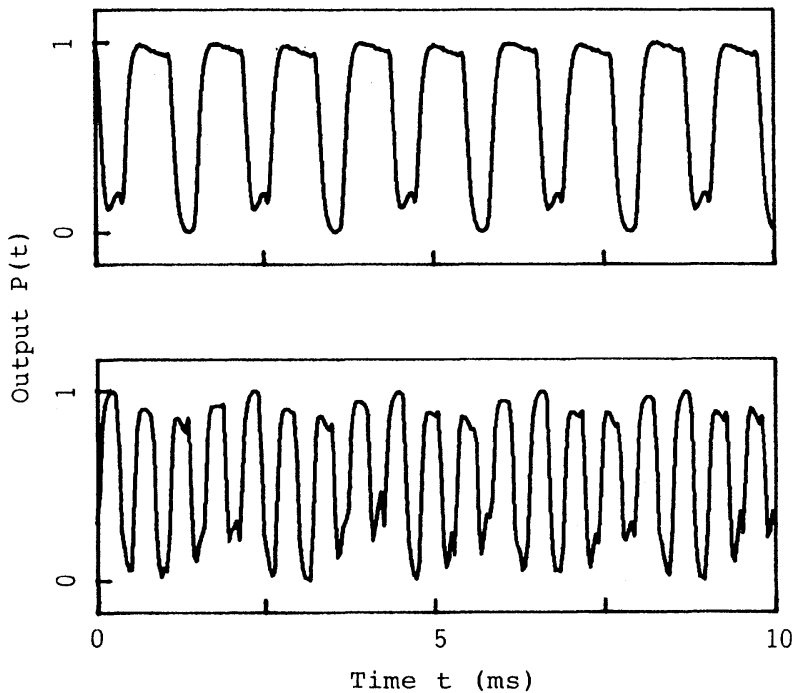

(a)

FIG. 9. Experimental results of the second-mode solution of spiking oscillations at the delay time of $1 \mathrm{~ms}$. (a) The fundamental solution and (b) the second-mode solution.

merical simulations, we used randomly distributed numbers as initial values of $P(t)$ within the first time interval $\left[0, t_{d}\right]$. The first several thousand steps for the length $t_{d}$ were discarded in the calculation to eliminate the longlived transient phenomena. In fact, the length of $2000 t_{d}$ for the precalculations was long enough to obtain the steady-state solutions. In the calculations, we assumed the value of $x(t)$ to be invariant at each time increment $\Delta t$ [typically one-hundredth of the damping time of $x(t)$, i.e., $\left.\gamma_{t} / 100\right]$ and solved Eqs. 2(a) and 2(b) for the fixed value of $x(t)$. By applying this approximation, we could reduce the computation time to a great extent. Such an approximation is appropriate, since the damping rates of $P(t)$ and $n(t)$ are larger enough than that of $x(t)$. We have actually verified that $P(t)$ and $n(t)$ always reach steady states within a time range of one-thousandth of the damping time of $x(t)$.

At the same parameter condition in Fig. 5, we calculated the wave forms of the output power for several different delay times. Figure 10 shows three examples of the stable wave forms obtained numerically for the cases of the single equilibrium shown in Figs. 5(a) and 5(c). At a small delay time, as plotted in the upper figure, the wave form behaves as a sinusoidal oscillation. At an increase of the delay time, the wave form becomes squaring and the amplitude increases. The same type of evolution has been described in Refs. [8] and [9]. The wave form bifurcates into subharmonic periodic cycles within the range of a moderate delay time. An example of period-4 cycles is shown in the middle of Fig. 10(a), while an example of period-3 cycles is shown in the middle of Fig. 10(b). With a further increase of the delay time, the wave form evolves into a chaotic region, as shown in the bottom of Fig. 10.

Figure 11 displays some numerical results of the wave forms at multiequilibria, such as those shown in Fig. 5(b). 

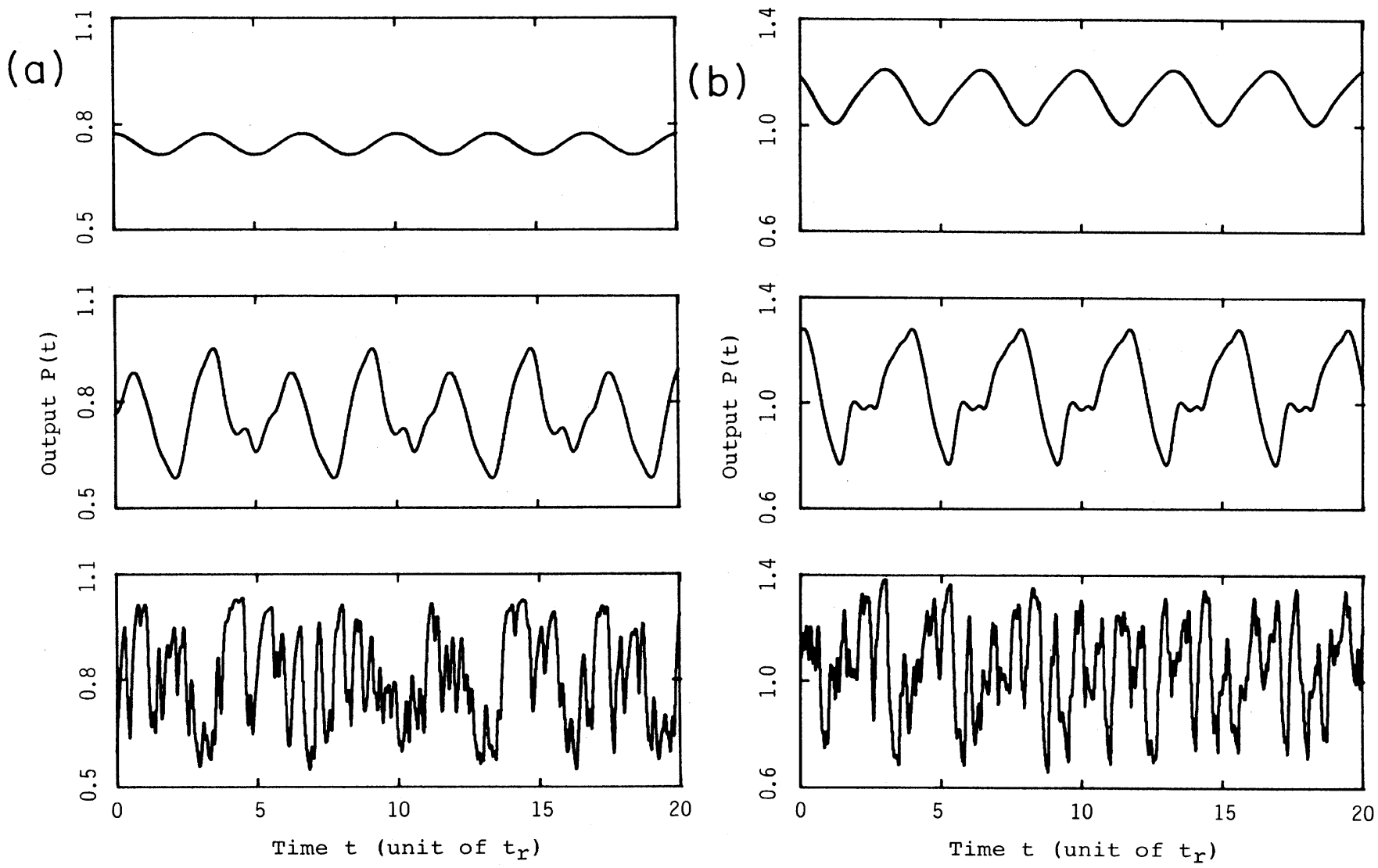

FIG. 10. Numerically calculated wave forms. (a) $t_{d}=0.5,1.5$, and $10.0 \gamma_{t}$ from top to bottom at $\bar{P}=0.74 \mathrm{~mW}$ and $j_{b}=1.27$. (b) $t_{d}=0.5,1.5$, and $10.0 \gamma_{t}$ from top to bottom at $\bar{P}=1.13$ and $j_{b}=1.37$.

The upper part of Fig. 11(a) displays a sinusoidal oscillation at the delay time of $10 \mu \mathrm{s}$. At a small delay time, the wave form resembles that for the case of a single equilibrium. This solution corresponds to that of the neighborhood point at the lower equilibrium in Fig. 5(b). An example of deformed sinusoidal oscillations at a rather small delay time of $20 \mu \mathrm{s}$ is shown in the middle of Fig. 11(a). It is also recognized that this solution corresponds to that of the neighborhood point at the upper equilibrium in Fig. 5(b). At a moderate delay time, the output power covers all of the three equilibria in Fig. 5(b) and the behavior of the wave form becomes different from that for the case of a small delay time. The bottom of Fig. 11(a) shows a solution obtained at a moderate delay time (typically $t_{d}=40 \sim 80 \mu \mathrm{s}$ ). The output power ranges from 0.7 to $1.2 \mathrm{~mW}$, which covers all of the three equilibria $(0.78,0.95$, and $1.09 \mathrm{~mW})$ in Fig. 5(b). The wave form behaves as a relaxationlike oscillation with two different periodic components. The envelope of the wave form has a period about ten times larger than the delay time, while the fine structure has a period near the delay time. With a further increase of the delay time, the period of the envelope increases, but the period of the fine structure changes little. In Fig. 11(b), three examples of the spiking oscillations are demonstrated at larger delay times. The figure shows the fundamental-, second-, and third-mode oscillations. The corresponding periods are $1.04,0.52$, and $0.34 t_{d}$, respectively. In the theoretical derivations, we have made several approximations in numerical simulation and neglected the temperature fluctuation of the laser diode and the effects of the spontaneous emission. In spite of such simplifications, the qualitative agreement between the numerical and experimental results is satisfactory.

\section{SUMMARY}

We have investigated theoretically and experimentally a new type of oscillations of the laser output power in a delayed-feedback bistable system. Such novel oscillations are characterized by a spiking feature of the wave form which has a period nearly equal to the delay time. The linear stability analysis has been carried out. The obtained mode distributions agree well with the spectral properties of the wave forms in the experiment. The numerical simulations reveal that such novel oscillations indeed occur under appropriate parameter conditions, including a proper delay time.

Recently, Otsuka and Chern have predicted theoretically a spiking pulse generation in a semiconductor laser 

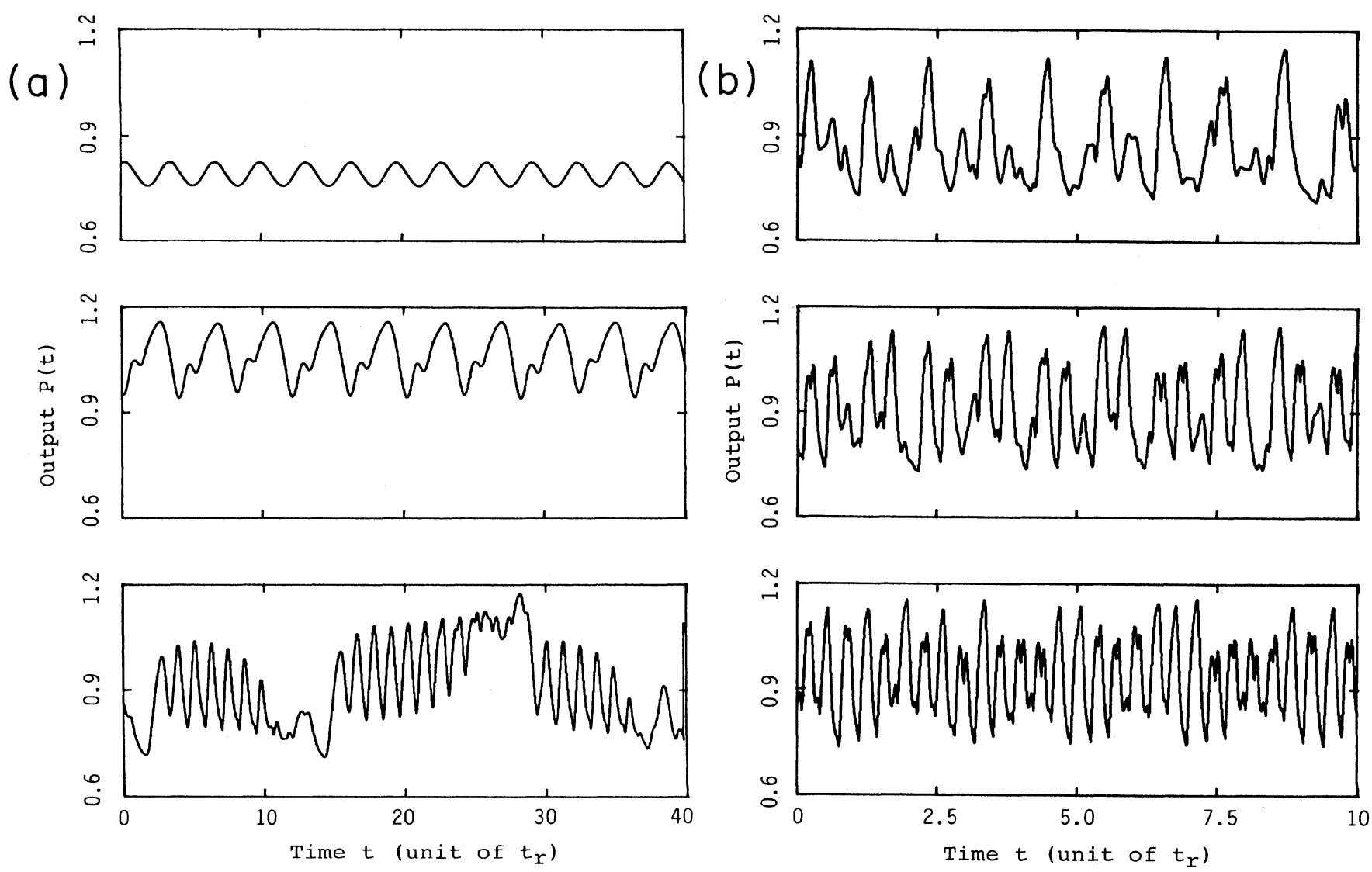

FIG. 11. Numerically calculated wave forms. (a) $t_{d}=0.5,1.0$, and $2.5 \gamma_{t}$ from top to bottom at $j_{b}=1.32$. (b) $t_{d}=10.0,13.0$, and $15.0 \gamma_{t}$ from top to bottom at $j_{b}=1.32$.

with an incoherent delayed optical feedback [17]. They treated the problem for a short delay time compared with the system response. On the other hand, in our case, we have shown that the spiking oscillations in a moderate region of the delay time result in different dynamics from, e.g., delay-time-locking and higher-mode solutions. Obviously, such oscillations with the same period of the delay time do not belong to the counterpart described by the discrete mappings. It is noted that this phenomenon would imply that the coupling between the discrete property and the continuous property of the actual physical system may lead to a very complicated dynamical behavior of the system output.

\section{ACKNOWLEDGMENT}

The work is supported by The Mechanical Industry Development and Assistance Foundation (MIDAF).

M. Yuan, ibid. 30, 901, (1984).

[1] Instability and Chaos in Quantum Optics, edited by F. T. Arecchi and R. G. Harrison (Springer-Verlag, Berlin, 1987).

[2] H. Haken, Rev. Mod. Phys. 47, 67 (1975).

[3] E. N. Lorenz, J. Atmos. Sci. 20, 130 (1963).

[4] K. Ikeda, Opt. Commun. 30, 257 (1979).

[5] H. M. Gibbs, F. A. Hopf, D. L. Kaplan, and R. L. Showmaker, Phys. Rev. Lett. 46, 474 (1981); F. A. Hopf, D. L. Kaplan, H. M. Gibbs, and R. L. Shoemaker, Phys. Rev. A 25, 2172 (1982).

[6] H. Nakatsuka, A. Asaka, H. Itoh, K. Ikeda, and M. Matsuoka, Phys. Rev. Lett. 50, 109 (1983).

[7] K. Ikeda, K. Kondo, and O. Akimoto, Phys. Rev. Lett. 49, 1467 (1982).

[8] J. Y. Gao, L. M. Narducci, L. S. Schulman, M. Squicciarini, and J. M. Yuan, Phys. Rev. A 28, 2910 (1983); J. Y. Gao, L. M. Narducci, H. Sadiky, M. Squicciarini, and J.
] P. Nardone, P. Mandel, and R. Kapral, Phys. Rev. A 33, 2465 (1986).

[10] M. Okada and K. Takizawa, IEEE J. Quantum Electron. QE-17, 2135 (1981).

[11] F. T. Arecchi, G. Giacomelli, A. Lapucci, and R. Meucci, Phys. Rev. A 43, 4997 (1991).

[12] Y. Liu and J. Ohtsubo, Opt. Commun. 85, 457 (1991); 93, 311 (1992).

[13] T. H. Dai, S. W. Hu, H. Tang, W. Deng, and H. T. Zang, Opt. Lett. 16, 899 (1991).

[14] J. Ohtsubo and Y. Liu, SPIE Proc. 1319, 50 (1990); Y. Liu and J. Ohtsubo, J. Opt. Soc. Am. B 9, 261 (1992).

[15] Y. Liu and J. Ohtsubo, Tech. Dig. 16, 325 (1992).

[16] R. Vallée and C. Delisle, Phys. Rev. A 31, 2390 (1985); 34, 309 (1986).

[17] K. Otsuka and J. L. Chern, Opt. Lett. 16, 1759 (1992). 